\title{
Segurança e eficácia do meloxicam associado à dipirona no tratamento da dor pós-operatória em cães*
}

\section{Safety and effectiveness of meloxicam associated to dipyrone in the treatment of post-surgical pain in dogs}

\author{
Jennifer Cristina Biscarra Bellio, ${ }^{* *}$ Maria Angélica Baron Magalhães, ${ }^{* *}$ Christiane Neves Garcia Pareja, ${ }^{* * * *}$ \\ Rita Maria Venâncio Mangrich Rocha, ${ }^{* * * * *}$ Pedro Vicente Michelotto Junior, ${ }^{* * * * * *}$ José Ademar Villanova Júnior, ${ }^{* * * * * *}$ \\ Cláudia Turra Pimpão********
}

\begin{abstract}
Resumo
O objetivo desta pesquisa foi avaliar a segurança e eficácia da associação de dois fármacos em um único medicamento à base de meloxicam e dipirona no tratamento da dor em cães. Primeiramente, avaliou-se a segurança do medicamento em 24 cães, distribuídos em três grupos $(n=8)$. Para avaliação da eficácia, foram estudadas 36 cadelas, submetidas a operações eletivas de ovariectomia $(\mathrm{OH})$, distribuídas em três grupos $(n=12)$. Nas duas etapas, o tratamento instituído foi o mesmo, e consistiu em: Grupo 1 - meloxicam 0,1 mg/kg um vez ao dia (SID); Grupo 2 - meloxicam 0,1 mg/kg + dipirona 25mg/kg SID e Grupo 3 - meloxicam $0,1 \mathrm{mg} / \mathrm{kg}$ + dipirona $25 \mathrm{mg} / \mathrm{kg}$ duas vezes ao dia (BID), sendo todos administrados por via oral. Na primeira etapa, os cães foram medicados por 28 dias consecutivos e exames hematológicos, urinálises e exames físicos foram realizados para avaliar possíveis efeitos colaterais das medicações. Na segunda etapa, as cadelas foram medicadas por cinco dias. A dor foi avaliada com base na escala de Glasgow e em exames físicos. Na primeira etapa, não houve diferença entre os grupos em relação aos exames laboratoriais. Não foram observadas alterações clínicas nos grupos 1 e 3, porém, em dois animais do Grupo 2, ocorreram vômito e diarreia. Na segunda etapa, não houve diferença entre os grupos em relação aos parâmetros clínicos e entre os escores de dor. A associação de meloxicam e dipirona, na dose de 0,1 mg/kg e 25mg/kg, respectivamente, a cada 12 ou 24 horas, mostrou-se segura e eficaz no controle da dor pós-operatória em cães.
\end{abstract}

Palavras-chave: AINEs, analgésico,efeitos adversos, eficácia, segurança.

\begin{abstract}
The present study aims to evaluate safety and effectiveness of a drug based on meloxicam associated to dipyrone. Animals were divided in three groups: Group 1 - meloxicam 0,1mg/kg, SID; Group 2 - meloxicam 0,1mg/kg + dipirona 25mg/kg, SID; Group 3 - meloxicam $0,1 \mathrm{mg} / \mathrm{kg}+$ dipirona $25 \mathrm{mg} / \mathrm{kg}$, BID. To evaluate the clinical and laboratorial safety, 24 healthy adult dogs were treated orally for 28 consecutive days. Physical examines and blood and urine collections were made to valuate de presence of side effects of the drugs. To evaluate the effectiveness in controlling $\mathrm{OH}$ postoperative pain, 36 bitches were treated orally for five days. Pain assessment by the criteria of the Glasgow pain scale of canine pain and clinical exams were performed daily. In the first part of the survey, there was no laboratorial difference between groups. No clinical alteration was noticed in groups 1 and 3 , but in Group 2 there were vomit and diarrhea en two animals. In the second part of the survey, there was no difference between clinical parameters and pain scores within each group, and no difference was found between groups in the physical examines. The association of meloxicam and dipyrone in dose of $0,1 \mathrm{mg} / \mathrm{kg}+25 \mathrm{mg} / \mathrm{kg}$, respectively, each 12 or 24 hours for up to 28 days, is safe and effectiveness in controlling post-surgical pain in dogs.
\end{abstract}

Keywords: analgesic, effectiveness, NSAIDs, safety, side effects.

\section{Introdução}

Dentre as opções para o controle farmacológico da dor estão os fármacos anti-inflamatórios não esteroidais (AINEs), como o meloxicam e a dipirona (Imagawa et al., 2011). Esses medicamentos apresentam bom efeito analgésico e anti-inflamatório, podendo ser utilizados de forma isolada ou associados a outros medicamentos (Caulkett et al., 2003; Imagawa et al., 2011).

O meloxicam é um AINE com propriedades antipirética e analgésica usado no tratamento de afecções musculoesqueléticas e no controle da dor pós-operatória. Na dose recomendada de $0,1 \mathrm{mg} / \mathrm{kg}$, apresenta atividade inibidora seletiva da enzima

*Recebido em 6 de fevereiro de 2015 e aceito em 4 de setembro de 2015.

${ }^{* *}$ M.V., Mestre em Ciência Animal pela Pontifícia Universidade Católica do Paraná (PUCPR)- Curitiba, PR

${ }^{* * *}$ M.V., MSc PhD, Pós-Doutorando em Ciência Animal pela Pontifícia Universidade Católica do Paraná (PUCPR) - Curitiba, PR

${ }^{* * * *}$ M.V., Médica Veterinária Residente de Anestesiologia na Pontifícia Universidade Católica do Paraná (PUCPR) - Curitiba, PR

*****M.V., MSc, Professora Adjunta de Patologia Clínica na Pontifícia Universidade Católica do Paraná (PUCPR) - Curitiba, PR

***** M.V., MSc, PhD, Professor Adjunto de Semiologia Clínica na Pontifícia Universidade Católica do Paraná (PUCPR) - Curitiba, PR

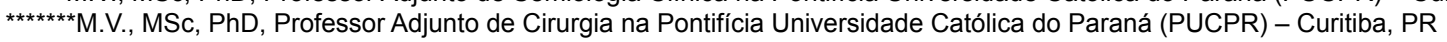

*******M.V., MSc, PhD, Professora Titular de Farmacologia e Toxicologia Veterinária na Pontifícia Universidade Católica do Paraná (PUCPR) - Curitiba, PR. Autor para correspondência: claudia.pimpao@pucpr.br 
cicloxigenase-2 (COX-2), mediador químico que desencadeia o processo inflamatório. Por causar baixa inibição da COX-1, seus efeitos colaterais são mínimos, e caracterizam-se geralmente por vômito e diarreia (Tasaka, 2011). É rapidamente eliminado do organismo, mas persiste por mais tempo em tecidos inflamados (Brune e Furst, 2007).

A dipirona é um AINE com ação analgésica e antipirética que pode eventualmente causar alterações gastrintestinais. Quando associada a outros AINEs, promove bom efeito analgésico com baixo risco de sangramento gastrointestinal (De Abajo, 2011).

Os principais efeitos adversos dos AINEs decorrem de toxicidade ao sistema digestório e caracterizam-se por vômito, diarreia, inapetência e, em casos severos, por perfuração de úlcera duodenal, com consequente peritonite, hepatoxicidade e morte (Tasaka, 2011).

Tanto a dipirona como o meloxicam são amplamente prescritos no tratamento da dor e inflamação de cães submetidos a procedimentos cirúrgicos. A associação dos dois fármacos em apenas um medicamento poderia facilitar sua administração aos animais, possibilitando um melhor tratamento pós-operatório.

O objetivo desta pesquisa foi avaliar a segurança e eficácia da associação de dois fármacos em um único medicamento à base de meloxicam e dipirona para o controle da dor em cães.

\section{Materiais e método}

Este trabalho seguiu as normas técnicas de pesquisa e experimentação animal (Petroianu, 1996; Petroianu, 2000) e foi realizado de acordo com a Lei 11.794 , de 8 outubro de 2008, nas dependências da Pontifícia Universidade Católica do Paraná (PUCPR) e recebeu aprovação do Comitê de Ética no Uso de Animais (CEUA) da PUCPR, sob parecer número 702.

Para a execução da pesquisa, foram estudados 60 cães adultos e hígidos, sendo 24 na primeira etapa e 36 na segunda, conforme descrição a seguir. Todos os cães foram mantidos em canis individuais, alimentados com ração comercial e receberam água ad libitum.

Para atender aos objetivos propostos, a presente pesquisa foi realizada em duas etapas.

A primeira etapa consistiu em um ensaio clínico duplo cego randomizado e teve por objetivo avaliar a segurança clínica e laboratorial de um medicamento à base de meloxicam associado à dipirona. Para isso, foram estudados 24 cães adultos hígidos, SRD, machos e fêmeas, de peso $16,30 \pm 3,98 \mathrm{~kg}$, provenientes do canil do Hospital Veterinário da PUCPR. Os animais foram distribuídos aleatoriamente em três grupos (Grupos 1A, 2A, 3A), com oito animais em cada:

Grupo 1A: meloxicam ${ }^{1} 0,1 \mathrm{mg} / \mathrm{kg}$ a cada 24 horas (SID), por via oral;

Grupo 2A: meloxicam 0,1 $\mathrm{mg} / \mathrm{kg}$ + dipirona $25 \mathrm{mg} / \mathrm{kg}$ a cada 24 horas (SID), por via oral;

Grupo 3A: meloxicam 0,1 mg/kg + dipirona $25 \mathrm{mg} / \mathrm{kg}$, a cada 12 horas (BID), por via oral.

O tratamento foi realizado por 28 dias consecutivos.

\footnotetext{
$\overline{{ }^{1} \text { Meloxivet } \circledast \text {, Duprat, }}$ Rio de Janeiro, RJ.
}

Em todos os animais dos três grupos, no primeiro dia de tratamento, o meloxicam foi administrado a cada 12 horas, na dose de $0,1 \mathrm{mg} / \mathrm{kg}$, conforme posologia preconizada (Gruet et al., 2011). Nos dias subsequentes, foram administradas as doses relacionadas para cada grupo.

Sete dias antes de iniciar a pesquisa, foi realizado exame clínico minucioso em cada animal, com vista a detectar quaisquer sinais de enfermidades. Além disso, exames hematológicos foram coletados por meio de punção venosa em todos os cães para realização de hemograma completo, TGP, TGO, FA, GGT, albumina, glicemia, creatinina e ureia. Foi coletada urina por meio de cistocentese guiada por ultrassonografia para urinálise. Os tubos contendo as amostras foram encaminhados ao laboratório de análises clínicas do Hospital Veterinário da PUCPR e imediatamente processadas para realização dos exames citados.

O exame físico dos animais incluiu avaliação do estado geral e da hidratação a partir da elasticidade da pele e do ressecamento das mucosas; do sistema cardiovascular (tempo de preenchimento capilar, frequência cardíaca e coloração de mucosas); do sistema respiratório (frequência respiratória e qualidade do som à ausculta); temperatura corporal e peso. Além disso, foram avaliados parâmetros relacionados ao trato gastrintestinal, como apetite, característica das fezes, presença ou ausência de vômito e sensibilidade à palpação abdominal.

Os exames clínicos de todos os cães foram realizados sete dias antes de iniciar a pesquisa e depois repetidos diariamente, sempre no mesmo horário, a partir do primeiro dia de estudo, ao longo dos 28 dias de acompanhamento. As análises laboratoriais, realizadas sete dias antes da pesquisa, foram repetidas nos dias 0,15 e 28 , após início do estudo.

Os dados foram apresentados em média e desvio padrão da média. Para verificar a normalidade dos dados, foi utilizado o teste de Kolmogorov-Smirnov. Os dados paramétricos foram comparados pela análise de variâncias (ANOVA), seguido do teste de Bonferroni. A verificação dos dados não paramétricos foi feita pelo teste de Kruskal Wallis. Todos os resultados foram considerados significativos para uma probabilidade de significância de pelo menos 95\% ( $p \leq 0,05)$. Os testes foram realizados utilizando o software GraphPad Prism 5.0, Califórnia, EUA.

A segunda etapa da pesquisa consistiu em um ensaio clínico duplo cego randomizado, cujo objetivo foi avaliar a eficácia da associação de dois fármacos em um único medicamento à base de meloxicam e dipirona, o mesmo testado na primeira etapa, no controle da dor e inflamação pós-operatórias em cadelas submetidas a ovário-histerectomia $(\mathrm{OH})$.

Para isso, foram estudadas 36 cadelas hígidas, adultas, SRD, provenientes do canil do Hospital Veterinário da PUCPR e da Sociedade Protetora dos Animais de Curitiba.

Os animais foram distribuídos aleatoriamente em três grupos (grupos 1B, 2B e 3B), com 12 animais cada:

Grupo 1B: meloxicam² $0,1 \mathrm{mg} / \mathrm{kg}$ SID, por via oral;

Grupo 2B: meloxicam 0,1 mg/kg + dipirona 25mg/kg SID, por via oral;

Grupo 3B: meloxicam 0,1mg/kg + dipirona 25mg/kg, BID, por via oral.

$\overline{2}$ Meloxivet $囚$, Duprat, $R$ io de Janeiro, RJ. 
Todas as cadelas foram submetidas a operações de $\mathrm{OH}$ eletiva.

Ultrassonografias abdominais foram realizadas antes do procedimento cirúrgico a fim de descartar doenças pré-existentes ou gestação.

Foi realizado jejum hídrico e alimentar de 12 horas antes do procedimento cirúrgico.

A técnica cirúrgica empregada seguiu os padrões de Fossum (2013).

Em todos os animais dos três grupos, no primeiro dia de tratamento, o meloxicam foi administrado a cada 12 horas, na dose de $0,1 \mathrm{mg} / \mathrm{kg}$, conforme posologia preconizada (Gruet et al., 2011). Nos dias subsequentes, foram administradas as doses relacionadas para cada grupo.

Durante o período cirúrgico, todas as cadelas receberam antibioticoprofilaxia à base de penicilina procaína e benzatina associada à di-hidroestreptomicina ${ }^{3}$, na dose de 8.000 a $24.000 U \mathrm{U} / \mathrm{kg}$, por via intramuscular (IM), em dose única.

Para evitar que os animais tivessem acesso à área operada, eles foram mantidos com colar elizabetano até a total cicatrização da ferida cirúrgica.

Durante o período pós-operatório, os animais foram avaliados clinicamente por cinco dias consecutivos, uma vez ao dia, sempre no mesmo horário, por um médico- veterinário que não tinha conhecimento sobre o tratamento instituído (avaliação duplo-cego). Para tanto, foram avaliados temperatura corporal (TC), frequência cardíaca (FC), frequência respiratória (FR) e tempo de preenchimento capilar (TPC), hidratação e coloração de mucosas. A hidratação foi mensurada por meio da elasticidade da pele e ressecamento de mucosas.

A dor foi avaliada por meio do formulário adaptado a partir da escala de dor de Glasgow (Tranquili et al., 2013). Esta ferramenta foi desenvolvida para mensurar a dor aguda em cães de forma psicométrica, que se baseia no comportamento dos cães e gera escores, permitindo uma avaliação objetiva e a posterior análise estatística (Murrel et al., 2008). Esse questionário é composto de seis seções, cuja somatória dos escores varia de 0 a 24 , em que 0 indica ausência total de dor e 24 a pior dor possível. Valores de 0 a 5 são sugestivos de ausência de dor (0) ou dor leve; 5 a 15 de dor moderada; 15 a 20 de dor intensa e valores de 20 a 24 indicam dor muito intensa (Coutinho, 2012).

A avaliação da dor foi feita diariamente, durante cinco dias, a partir do dia do procedimento cirúrgico, obedecendo a cuidados como não usar jaleco ou vestimenta cirúrgica, não portar ração, não fazer afagos nem tratar os animais pelos nomes para não comprometer os resultados. Essas avaliações foram realizadas por um médico-veterinário sem o conhecimento prévio do tratamento instituído, o qual dava início à aproximação do canil observando o comportamento e as reações de cada animal ainda do lado de fora.

Para a análise dos dados, foi utilizado o teste Kruskal-Wallis seguido de teste Dunn's. Os dados são apresentados em medianas. O nível de significância adotado foi $5 \%(\alpha=0,05)$. Todos os cálculos foram realizados utilizando-se o Software estatístico GraphPad Prism version 5.00 for Windows, San Diego - Califórnia, EUA.

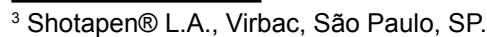

\section{Resultados e discussão}

Animais submetidos a $\mathrm{OH}$ apresentam dor de intensidade moderada no pós-operatório, decorrente de trauma, manipulação e inflamação tecidual, e por isso requerem protocolo analgésico adequado (Slingsby et al., 2011). A classe de medicamentos mais utilizada nessa situação é a dos AINEs, devido a seu fácil acesso, baixo custo e sua eficácia. Porém, sabe-se que a associação de diferentes tipos de medicamentos pode beneficiar o paciente, por promover melhor controle da dor e diminuição dos efeitos colaterais. Diante disso, foi desenvolvido um comprimido utilizando-se a formulação de $1 \mathrm{mg}$ de meloxicam associado a $250 \mathrm{mg}$ com dipirona, o que equivale a um comprimido para cada $10 \mathrm{~kg}$ de peso corporal.

O meloxicam é um AINE com conhecido efeito analgésico. A dipirona é um fármaco com pouca ação anti-inflamatória, porém, com potentes efeitos analgésico e antipirético. Esses dois fármacos são amplamente utilizados na rotina médica veterinária devido a sua segurança e eficácia (Brune e Furst, 2007), além de seu baixo preço. A formulação de um único medicamento contendo dois princípios ativos eficazes e seguros constitui um bom recurso para tratar a dor de cães no pós-operatório.

No intuito de facilitar a administração da dipirona e do meloxicam, minimizando o estresse sofrido pelo animal durante a medicação, no presente estudo, os dois princípios ativos foram formulados em um só comprimido, o qual foi dado por via oral, sem resistência por parte dos animais.

Todos os animais do estudo apresentaram estado geral bom e mucosas normocoradas ao longo de todo o período de observação. Além disso, o apetite, bem como o grau de hidratação de todos eles, mantiveram-se estáveis.

Não houve diferença dentro dos grupos e entre os mesmos $(p>0,05)$ em relação aos valores de FC, FR, TC, TPC e peso nas duas etapas da pesquisa.

Em relação aos possíveis efeitos adversos que poderiam surgir devido ao uso dos medicamentos testados, não foram observadas complicações nos animais dos grupos $1 \mathrm{Ae} 3 \mathrm{~A}$. No Grupo 2A, foi observada êmese e hiporexia em dois cães (16\%), sendo um no sétimo e outro no décimo quinto dia de acompanhamento. Diarreia pastosa acompanhada de sensibilidade abdominal ocorreu em um cão desse mesmo grupo entre o décimo terceiro e o décimo sexto dias de acompanhamento. O medicamento não foi suspenso nesses casos e todos os animais recuperaram-se espontaneamente, sem necessidade de intervenções.

Acredita-se que as alterações observadas no Grupo 2A podem estar associadas aos efeitos colaterais conhecidos do meloxicam e da dipirona. Porém, essas reações não comprometeram o estado clínico dos animais, já que elas foram leves e cederam espontaneamente, sem necessidade de outras medicações.

Os efeitos colaterais mais frequentes do meloxicam incluem problemas gastrintestinais, como gastrite, náuseas, vômitos e diarreia (Singh et al., 1998; Lamont e Mathews, 2008). Em um estudo realizado por Hare et al. (2012) com 40 cães, em que o meloxicam foi administrado por via transmucosa por meio de spray oral de uma a cinco vezes ao dia, durante seis meses, foram observadas complicações como diarreia, êmese, alterações na consistência fecal, melena, alterações urinárias e comportamentais. Alguns desses achados foram semelhantes aos encontrados em nosso estudo, porém, na presente pesquisa, 
observaram-se menos efeitos colaterais, provavelmente em decorrência do menor tempo de tratamento com meloxicam, quando comparado ao estudo de Hare et al. (2012).

Luna et al. (2007) analisaram os efeitos adversos do uso prolongado de diferentes AINEs por 90 dias, como carprofeno, flunixin meglumine, cetoprofeno e meloxicam, administrados por via oral. Concluíram que o meloxicam foi o mais seguro em relação a alterações gastrintestinais, uma vez que causou menor frequência de efeitos adversos nos animais estudados. Esses achados vão de encontro ao presente estudo, uma vez que o medicamento foi bem tolerado, mesmo pelos animais que o receberam duas vezes ao dia.

Um trabalho realizado por Imagawa et al. (2011) mostrou que, na dose de 25 a $35 \mathrm{mg} / \mathrm{kg}$, por via intravenosa, a dipirona é capaz de provocar êmese durante as primeiras seis horas de pós-operatório, regredindo espontaneamente. Esse fato não foi observado na presente pesquisa, o que confirma a segurança clínica do fármaco. Essas reações geralmente são individuais, não ocorrendo com frequência na rotina clínica cirúrgica.

Em relação aos valores de eritrócitos, hemoglobina, hematócrito, leucócitos totais e plaquetas, não foi observada diferença dentro de cada grupo e entre os grupos estudados na primeira etapa $(1 \mathrm{~A}, 2 \mathrm{~A}$ e $3 \mathrm{~A})(\mathrm{p}>0,05)$.

Não foi observada diferença em relação aos valores hematológicos em nenhum momento do experimento. Este fato reforça a segurança clínica dos medicamentos testados. Dentre os efeitos adversos provocados pelo meloxicam, podem ser observadas alterações no processo de hemostasia, o que não parece ter ocorrido em nosso trabalho, corroborando os resultados de Mathews et al. (2001), Caulkett et al. (2003) e Fresno et al. (2005). Esses autores demostraram que, quando administrado em dose única de $0,2 \mathrm{mg} / \mathrm{kg}$, por via parenteral, o meloxicam não compromete a hemostasia de cadelas saudáveis submetidas à $\mathrm{OH}$ eletiva e operações abdominais. Outro estudo realizado em 2010, por Blois e colaboradores, mostrou que a administração oral de meloxicam na dose $0,1 \mathrm{mg} / \mathrm{kg}$ a cada 24 horas, durante sete dias, não alterou a função plaquetária. Esses resultados corroboram os obtidos na presente pesquisa, em que a administração de meloxicam associado à dipirona não alterou o número de plaquetas em todos os animais do estudo. Contudo, Luna et al. (2007) reportaram tempo de sangramento prolongado em cães submetidos a tratamento com meloxicam, por sete dias, na dose $0,1 \mathrm{mg} / \mathrm{kg}$ a cada 24 horas, por via oral, o que não foi avaliado no presente estudo.

A administração isolada de dipirona não foi associada a alterações nos parâmetros laboratoriais (Mathews, 2000; Flôr et al., 2013). Entretanto, quando associada a AINEs, pode relacionar-se a sangramento gastrintestinal (De Abajo, 2011). Imagawa et al. (2011) mostraram que, na dose de 25 a $35 \mathrm{mg} / \mathrm{kg}$, por via intravenosa, a dipirona pode provocar algumas discrasias sanguíneas. Entretanto, não foram observadas alterações laboratoriais nos animais em nossa pesquisa.

Não foi observada agranulocitose no estudo realizado por Flôr et al. (2013) com 69 cães medicados com dipirona associada a outros AINEs, na dose de $25 \mathrm{mg} / \mathrm{kg}$ a cada 12 horas, durante 14 dias, para o tratamento da dor oncológica. Esses dados corroboram os resultados encontrados em nosso estudo, uma vez que não foram observadas alterações na contagem de leucócitos em nenhum dos três grupos estudados.
Não foi encontrada diferença $(p>0,05)$ dentro dos grupos $1 A, 2 A$ e $3 \mathrm{~A}$, bem como entre os mesmos em relação aos parâmetros bioquímicos avaliados. A função renal dos animais na primeira etapa da pesquisa manteve-se estável, reforçando os estudos realizados por Borges et al. (2013), Fusellier et al. (2007) e Boström et al. (2006). Os AINEs inibem a síntese de PGs, podendo causar vasoconstrição renal e consequente diminuição da perfusão dos rins. Isso poderia resultar em redução da função, elevando os valores de ureia e creatinina séricos. Esse fato não foi observado neste estudo, sugerindo que não houve prejuízo à função renal nos animais. Resultados semelhantes foram encontrados por Imagawa et al. (2011), os quais mostraram que, nas doses de 25 a $35 \mathrm{mg} / \mathrm{kg}$, por via intravenosa, a dipirona não desencadeou alterações renais após dois dias de tratamento em cães.

Não foi observada alteração nos resultados de urinálise durante os 28 dias de avaliação $(p>0,05)$. Esses achados são compatíveis com os dados de função renal encontrados nos exames bioquímicos deste estudo, que demonstram não terem ocorrido alterações de função renal nos animais tratados. Os resultados deste estudo corroboram os de Borges et al. (2013), que não encontraram alterações urinárias nos cães tratados com meloxicam na dose de $0,2 \mathrm{mg} / \mathrm{kg}$, uma vez ao dia, durante 10 dias.

Em relação à avaliação da eficácia do medicamento testado, não foram encontradas diferenças nos valores de FC, FR, TPC entre os grupos $1 B, 2 B$ e $3 B(p>0,05)$ e dentro dos mesmos, durante os cindo dias de tratamento, sugerindo que não houve manifestação de dor intensa pelas cadelas. Porém, houve aumento de TC $(p<0,05)$ dentro dos grupos $2 B$ e $3 B$, ao longo dos cinco dias de avaliação, mas os valores mantiveram-se dentro da normalidade. Esse pequeno aumento na TC pode ter ocorrido em função das altas temperaturas climáticas registradas na época do estudo e da agitação de algumas cadelas durante as avaliações clínicas.

Em relação à avaliação da dor segundo escala de Glasgow, não houve diferença nos valores dos escores de dor entre grupos $(p>0,05) 1 B, 2 B$ e 3B durante os cinco dias de tratamento.

No Grupo 1B, o valor mais alto atingido no somatório total de escores foi 5 . No Grupo 2B, o valor máximo atingido no somatório de escores foi 7 . Já no grupo 3B, o valor máximo atingido no somatório de escores foi 7 , porém a mediana dos 3 grupos estudados foi igual a 3 nos primeiros dois dias de avaliação, reduzindo a 1 no terceiro dia e 0 nos dois últimos dois dias de avaliação.

A avaliação do grau de dor mostrou que não houve diferença em relação ao efeito analgésico dos medicamentos entre os grupos. Apesar da semelhança entre os grupos, observou-se adequada analgesia em todas as cadelas, já que as mesmas apresentaram escore baixo na escala de Glasgow. Escores de 5 a 15 no somatório na escala de dor são considerados como dor moderada para cadelas submetidas a $\mathrm{OH}$ (Coutinho, 2012). $\mathrm{Na}$ presente pesquisa, todas as cadelas apresentaram valores de escore abaixo de 7 , sugerindo que o protocolo testado nessa pesquisa foi eficaz no controle da dor.

Aescala de Glasgow foi utilizada neste estudo por ser um método prático, fácil e eficaz e, de acordo com Holton et al. (2001), constitui a escala validada mais adequada para a avaliação da dor aguda pós-operatória em cães. 
Estudo realizado por Leece et al. (2005) apontou que o meloxicam na dose de $0,1 \mathrm{mg} / \mathrm{kg}$, a cada 24 horas, promoveu analgesia satisfatória por 72 horas após $\mathrm{OH}$ em cadelas, o que foi avaliado mediante escala analógica visual. Outro estudo demonstrou que esse medicamento é seguro e eficaz para controlar a dor pós-operatória por até 20 horas em cães que passaram por laparotomias (Mathews et al., 2001). Caulkett et al. (2003) afirmaram que o meloxicam produz efetiva ação analgésica em cães e gatos, porém depende da causa e tipo de dor, bem como, associações e doses. Pimpão et. al. (2009) afirmaram que os AINEs promovem melhor analgesia pós-operatória do que os opioides, além de possuírem efeito antipirético. Esses resultados reforçam a importância de se prescrever um AINE para o tratamento da dor pós-operatória em cães.

Em um estudo realizado por Martins et al. (2010), a dipirona na dose de $25 \mathrm{mg} / \mathrm{kg}$, por via oral, a cada 8 horas, por 10 dias, promoveu analgesia no período pós-operatório em 13 de 14 animais submetidos a manbibulectomias ou maxilectomias. Imagawa et al. (2011) mostraram que, na dose de $25 \mathrm{mg} / \mathrm{kg}$, a cada 8 horas, a dipirona promoveu analgesia satisfatória nos dois primeiros dias de pós-operatório de $\mathrm{OH}$. Após testar diferentes doses de dipirona (15mg/kg, 25mg/kg e $35 \mathrm{mg} / \mathrm{kg}$ ) para o controle da dor em cães, Bonagura (2000) observou que a dose apropriada é de $25 \mathrm{mg} / \mathrm{kg}$, a cada 12 horas, em concordância com o protocolo utilizado em nosso trabalho. Os dados dos trabalhos citados estão de acordo com os encontrados na presente pesquisa, já que a dipirona, na dose de $25 \mathrm{mg} / \mathrm{kg}$ uma

\section{Referências}

BLOIS, S.L.; ALLEN, D.G.; WOOD, R.D.; CONLON, P.D. Effects of aspirin, carprofen, deracoxib, and meloxicam on platelet function and systemic prostaglandin concentrations in healthy dogs. American Journal of Veterinary Research, v. 71, n. 5, p. 349-358, 2010.

BONAGURA, J.B. Table of common drugs: approximate dosages. In: IMAGAWA, V.H.; FANTONI, D.T.; TATARUNAS, A.C.; MASTROCINQUE, S.; ALMEIDA, T.F.; FERREIRA, F.; POSSO, I.P. The use of different doses of metamizol for post-operative analgesia in dogs. Veterinary Anaesthesia and Analgesia, v. 38, n. 4, p. 385-393, 2011.

BORGES, M.; MARINI, R.; LAPOSY, C.B.; GUIMARÃESOKAMOTO, P.T.C.; CHAVES, M.P.; L.E.; VIEIRA, A.N.; MELCHERT, A. Nonsteroidal anti-inflammatory therapy. Changes on renal function of healthy dogs. Acta Cirúrgica Brasileira, v. 28, n. 12, p. 843-847, 2013.

BOSTRÖM, I.M.; NYMAN, G.; HOPPE, A.; LORD, P. Effects of meloxicam on renal function in dogs with hypotension during anaesthesia. Veterinary Anaesthesia and Analgesia, v. 33, n. 1, p. 62-69, 2006.

BRUNE, K.; FURST, D.E. Combining enzyme specificity and tissue selectivity of cyclooxygenase inhibitors: towards better tolerability? Rheumatology, v. 46, n. 6, p. 911-919, 2007.

CAULKETT, N.; READ, M.; FOWLER, D.; WALDNER, C. A comparison of the analgesic effects of butorphanol with those of meloxicam after elective ovariohysterectomy in dogs. Canadian Veterinary Journal, v. 44, n. 7, p. 565-570, 2003.

COUTINHO, A. F. O. S. V. Subjetividade na avaliação da dor animal. 79f. Dissertação (Mestrado) - Faculdade de Medicina Veterinária - Universidade técnica de Lisboa, 2012. ou duas vezes ao dia, mostrou-se segura e eficaz no controle da dor pós-operatória de cadelas submetidas à $\mathrm{OH}$.

Apesar de, em nossa pesquisa, não ter havido diferença em relação ao efeito analgésico observado no grupo que recebeu apenas meloxicam, daquele que recebeu meloxicam mais dipirona, é sabido que muitos animais apresentam maior sensibilidade para dor e febre do que outros. Para muitos cães, a administração isolada de um AINE no pós-operatório não é suficiente para um adequado tratamento da dor. Portanto, a associação de um AINE a outro fármaco analgésico e antipirético poderia proporcionar um melhor efeito analgésico do que a administração isolada de um AINE.

Assim, a formulação de um único medicamento contendo dois princípios ativos eficazes e seguros constitui um bom recurso de adminstração para tratar a dor e a inflamação de cadelas no período pós-operatório.

\section{Conclusões}

A associação de meloxicam e dipirona, administrados por via oral, na dose de $0,1 \mathrm{mg} / \mathrm{kg}$ e $25 \mathrm{mg} / \mathrm{kg}$, respectivamente, a cada 24 ou a cada 12 horas, por até 28 dias, mostrou-se segura do ponto de vista clínico e laboratorial, pois não apresentaram efeitos adversos significativos. Com relação à avaliação da dor com a administração da formulação testadas por 5 dias, independentemente da dose utilizada, mostrou que a dipirona não promove um efeito aditivo para melhorar a analgesia no pós-operatório de cadelas submetidas a operações de $\mathrm{OH}$.

DE ABAJO, F.J. Effects of selective serotonin reuptake inhibitors on platelet function: mechanisms, clinical outcomes and implications for use in elderly patients. Drugs Aging, v. 28, n. 5, p. 345-367, 2011.

FLÔR, P.B.; YAZBEK, K.V.B.; IDA, K.K.; FANTONI, D.T. Tramadol plus metamizole combined or not with anti-inflammatory drugs is clinically effective for moderate to severe chronic pain treatment in cancer patients. Veterinary Anaesthesia and Analgesia. v. 40, n. 3, p. 316-327, 2013.

FOSSUM, T.W. Surgery of the reproductive and genital system. In: FOSSUM, T.W. Small animal surgery. St. Louis, 2013, p. 780-794.

FRESNO, L.; MOLL, J.; PEÑALBA, B.; ESPADA, Y.; ANDALUZ, A.; PRANDI, D.; DE GOPEGUI, R.R.; GARCÍA, F. Effects of preoperative administration of meloxicam on whole blood platelet aggregation, buccal mucosal bleeding time, and haematological indices in dogs undergoing elective ovariohysterectomy. The Veterinary Journal, v. 170, n.1, p. 138-140, 2005.

FUSELLIER, M.; DESFONTIS, J.C.; LE ROUX, A.; MADEC, S.; GAUTIER, F.; THULEAU, A.; GOGNY, M. Effect of short-term treatment with meloxicam and pimobendan on the renal function in healthy beagle dogs. Journal of Veterinary Pharmacology Therapy, v. 31, n. 2, p. 150-155, 2007.

GRUET, P.; SEEWALD, W.; KING, J.N. Evaluation of subcutaneous and oral administration of robenacoxib and meloxicam for the treatment of acute pain and inflammation associated with orthopedic surgery in dogs. American Journal of Veterinary Research, v. 72, n. 2, p. 184-193, 2011.

HARE, J.E.; NIEMULLER, C.A.; PETRICK, D.M. Target animal safety study of meloxicam administered via transmucosal oral spray (Promist $\circledast$ technology) for 6 months in dogs. Journal of Veterinary Pharmacology Therapy, v. 36, n. 4, p. 412-416, 2012. 
HOLTON, L.; REID, J.; SCOTT, E.M.; PAWSON, P.; NOLAN, A. Development of behavior-based scale to measure acute pain in dogs. Veterinary Research, v. 148, n.17, p. 525-531, 2001.

IMAGAWA, V.H.; FANTONI, D.T.; TATARUNAS, A.C.; MASTROCINQUE, S.; ALMEIDA, T.F.; FERREIRA, F.; POSSO, I.P. The use of different doses of metamizol for post-operative analgesia in dogs. Veterinary Anaesthesia and Analgesia, v. 38, n. 4, p. 385-393, 2011.

LAMONT, L.A.; MATHEWS, K.A. Opioids, nonsteroidal antiinflammatories and analgesic adjuvants. In: LUMB \& JONES. Veterinary Anesthesia and Analgesia. Blackwell Publishing, Ames, 2008, p. 241-271.

LEECE, E.A.; BREARLEY, J.C.; HARDING, E.F. Comparison of carprofen and meloxicam for 72 hours following ovariohysterectomy in dogs. Veterinary Anaesthesia and Analgesia, v. 32, n. 4, p. 184-192, 2005.

LUNA, S.P.L.; BASÍLIO, A.C.; STEAGALL, P.V.M.; MACHADO, L.P.; MOUTINHO, F.Q.; TAKAHIRA, R.K.; BRANDÃO, C.V.S. Evaluation of adverse effects of long-term oral administration of carprofen, etodolac, flunixin meglumine, ketoprofen, and meloxicam in dogs. American Journal of Veterinary Research, v. 68, n. 3, p. 258-264, 2007.

MARTINS, T.L.; KAHVEGIAN, M.N.; NOEL-MORGAN, J. Comparison of the effects of tramadol, codeine, and ketoprofen alone or in combination on postoperative pain and on concentrations of blood glucose, serum cortisol, and serum interleukin-6 in dogs undergoing maxillectomy or mandibulectomy. American Journal of Veterinary Research, v. 71, n. 9, p. 10191026, 2010.

MATHEWS, K.A. Pain assessment and general approach to management. Veterinary Clinics of North America: Small Animal Practice, v. 30, n. 4, p. 729-755, 2000.
MATHEWS, K.A.; PETTIFER, G.; FOSTER, R.; MCDONELL, W. Safety and efficacy of preoperative administration of meloxicam, compared with that of ketoprofen and butorphanol in dogs undergoing abdominal surgery. American Journal of Veterinary Research, v. 62, n. 6, p. 882-887, 2001.

MURRELL, J.C.; PSATHA, E.P.; SCOTT, E.M.; REID, J.; HELLEBREKERS, L.J. Application of a modified form of the Glasgow pain scale in a veterinary teaching centre in the Netherlands. Veterinary Record, v. 162, n. 13, p. 403-408, 2008.

PETROIANU, A. Aspectos éticos na pesquisa em animais. Acta Cirúrgica Brasileira. v. 11, p. 157-164, 1996.

PETROIANU, A. Pesquisa experimental. In: PETROIANU A, editor. Ética, Moral e Deontologia Médicas. Rio de Janeiro: Guanabara Koogan, 2000, p. 185-190.

PIMPÃO, C.T.; MONTANHA, F.P.; BUDZIAK, C. Avaliação do carprofeno e do meloxicam como antipirético em cães. Revista Acadêmica Ciências Agrárias e Ambientais, v. 7, p. 331-339, 2009.

SINGH, G.; RAMEY, D. R. NSAID induced gastrointestinal complications: The ARAMIS perspective-1997. Jounal of Rheumatology, v. 25, p. 8-16, 1998.

SLINGSBY, L.S.; TAYLOR, P.M.; MURRELL, J.C. A study to evaluate buprenorphine at $40 \mu \mathrm{g} \mathrm{kg}-1$ compared to $20 \mu \mathrm{g} \mathrm{kg}-1$ as a post-operative analgesic in the dog. Veterinary Anaesthesia and Analgesia, v. 38, n. 6, p. 584-593, 2011.

TASAKA, A.C. Anti-inflamatórios não esteroidais. In: SPINOSA, HS, GÓRNIAK, SL, BERNARDI, MM. Farmacologia Aplicada à Medicina Veterinária. 5. ed. Rio de Janeiro: Guanabara Koogan, 2011, p. 245-260.

TRANQUILI, W.J.; THURMON, J.C.; GRIMM, K.A. Anestesiologia e analgesia veterinária. 4. ed. São Paulo: Roca, 2013, 53 p. 